\title{
Grafting of calcium chelating functionalities onto PLA monofilament fiber surfaces
}

Cite as: Biointerphases 15, 011006 (2020); https://doi.org/10.1116/1.5129989

Submitted: 03 October 2019 . Accepted: 22 January 2020 . Published Online: 21 February 2020

Elias Mulky (D), Giuseppino Fortunato (D), Dirk Hegemann (D), Jorge Sague, Roman Heuberger (D), and Martin Frenz (D)

\section{ARTICLES YOU MAY BE INTERESTED IN}

Formation and characteristics of mixed lipid/polymer membranes on a crystalline surfacelayer protein lattice

Biointerphases 15, 011002 (2020); https://doi.org/10.1116/1.5132390

Polystyrene-block-polyethylene oxide thin films: In vitro cytocompatibility and protein adsorption testing

Biointerphases 15, 011003 (2020); https://doi.org/10.1116/1.5135062

Rapid detection of urokinase plasminogen activator using flexible paper-based graphene-gold platform

Biointerphases 15, 011004 (2020); https://doi.org/10.1116/1.5128889

\section{AVs Quantum Science}




\title{
Grafting of calcium chelating functionalities onto PLA monofilament fiber surfaces
}

\author{
Cite as: Biointerphases 15, 011006 (2020); doi: $10.1116 / 1.5129989$ \\ Submitted: 3 October 2019 . Accepted: 22 January 2020 . \\ Published Online: 21 February 2020
}

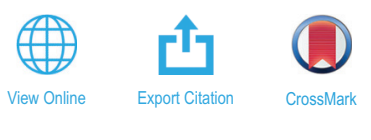

Elias Mulky, ${ }^{1}$ (D) Giuseppino Fortunato, ${ }^{2}$ (1) Dirk Hegemann, ${ }^{2}$ (D) Jorge Sague, ${ }^{7}$ Roman Heuberger, ${ }^{1, a)}$ (D) and Martin Frenz ${ }^{3, a)}$ (D)

\author{
AFFILIATIONS \\ ${ }^{1}$ RMS Foundation, Bischmattstrasse 12, Bettlach 2544, Switzerland \\ ${ }^{2}$ Empa, Swiss Federal Laboratories for Materials Science and Technology, Lerchenfeldstrasse 5, 9014 St Gallen, Switzerland \\ ${ }^{3}$ Institute of Applied Physics, University of Bern, Sidlerstrasse 5, 3012 Bern, Switzerland
}

a) Authors to whom correspondence should be addressed: roman.heuberger@rms-foundation.ch and frenz@iap.unibe.ch

\begin{abstract}
Polymer surface grafting is widely used in the field of bone regeneration to increase calcium phosphate (CaP) adhesion, with the intent of improving mechanical properties of CaP-polymer composite cements. Reinforcement can be achieved using multiple combined functional groups and/or complex surface geometries that, however, concurrently influence multiple effects such as wetting, roughness, and interfacial strengthening. This study focused on the influence of a chelating group, namely aspartic acid, on the adsorption of divalent ions such as $\mathrm{Ba}^{2+}$ or $\mathrm{Ca}^{2+}$ onto poly-L-lactic acid (PLA) films. The films were analyzed using contact angle measurements and X-ray photoelectron spectroscopy. The adsorption of $\mathrm{CaP}$ and its interfacial mechanical properties were investigated using functionalized PLA monofilaments whose surface roughness was analyzed using white light interferometry. Mechanical analysis was conducted by performing pull-out tests. The surfaces were analyzed using scanning electron microscopy and energy dispersive X-ray spectroscopy. Using aspartic acid as a chelating group resulted in a $50 \%$ increased adsorption of barium, an almost threefold increase in calcium coverage of the fiber compared to the control group and a twofold increase in interfacial stiffness. No significant increase in interfacial strength was determined, most likely due to the weakness of the $\mathrm{CaP}$ matrix, which was partially visible as residues on the monofilaments in the postfracture imaging. This study shows the potential of surfaces functionalized with aspartic acid as a simple alternative to complex polypeptide based functional groups for the adsorption of divalent ions such as calcium on poly-lactic acid in bone regenerating applications.
\end{abstract}

Published under license by AVS. https://doi.org/10.1116/1.5129989

\section{INTRODUCTION}

In the field of bone regeneration, calcium phosphate $(\mathrm{CaP})$ based cements are widely used due to their excellent biocompatibility and tunable characteristics such as geometry, strength, porosity, setting time, and time to total absorption. However, these $\mathrm{CaP}$ cements are generally brittle and of low mechanical strength due to the porous, disoriented polycrystalline nature of the set cement. ${ }^{1}$ Reinforcing these cements with polymer filaments can increase fracture strength and reduce the cement brittleness due to the combination of high polymer toughness with the rigidity of the cement phase. ${ }^{2}$ To allow for efficient stress transfer, a strong bond between the polymeric filament and the matrix interface is needed to avoid structural rigidity. ${ }^{3}$ In fact, an important mechanism contributing to the mechanical strength of bone is the presence of strong hydrogen bonds between the fibrous collagen phase and single crystalline hydroxyapatite (HA) platelets. ${ }^{4}$ Of particular interest for biodegradable cements are tough biodegradable polymers based on polyglycolic acid (PGA) or polylactic acid (PLA) with high tensile strengths in the order of $70 \mathrm{MPa}$ and stiffness around $2 \mathrm{GPa}$. For slow degrading cements and cements reacted using aqueous solutions, PLA is more suitable as a reinforcing agent compared to PGA. This is based on the slower degradation rate of PLA in aqueous media, meaning that the fibers can hold the mechanical integrity of the cement for a longer time. This stems from the higher hydrophobicity of PLA (static water contact angle of $70^{\circ}$ up to $120^{\circ}$ in electrospun nonwovens) compared to PGA (water contact angle of $\left.40^{\circ}-60^{\circ}\right),{ }^{5,6}$ However, the hydrophobic nature of PLA results in a poor interfacial bond with the hydrophilic $\mathrm{CaP}$ cement. ${ }^{7}$ To increase the adhesion strength between the CaP cement and PLA filaments, a suitable interlayer has to be introduced between the two phases. 
Many methods exist to manufacture the interlayer, such as dip and spin coating, plasma deposition, and wet chemical methods. The latter two are of particular interest since they modify the polymer surface chemically resulting in durable interfaces. In particular, plasma deposition can be performed as a continuous reel-to-reel process as demonstrated by Leal and co-workers with plasma activated continuous polyethylene filaments. ${ }^{8}$

This interlayer can either be engineered to match the surface energy of the $\mathrm{CaP}$ more closely, i.e., to increase the hydrophilicity of the PLA, or to specifically adsorb a component of the CaP cement. The first approach, namely, increasing the hydrophilicity of the PLA, can be achieved by depositing different hydrophilic groups onto the surface. For this purpose, the plasma deposition technique is widely used. ${ }^{9}$ Such studies were performed by Canal and co-workers ${ }^{10}$ who subjected chopped multifilamentous PLA to $\mathrm{O}_{2}$ plasma and mixed it with $\mathrm{CaP}$ to form a PLA-CaP cement. The plasma treatment resulted in an increase in both surface roughness and hydrophilicity of the treated PLA (static water contact angle of $62^{\circ}$ ). The interfacial strength was indirectly measured by conducting mechanical tests on the treated cements indicating an increase in the work of fracture of the cements containing the treated multifilaments, while the flexural strength remained similar to the pristine cement. This was attributed to the increased contact surface between the CaP and the PLA due to the increased hydrophilicity. Despite the increase in surface roughness resulting from the plasma treatment, the effect was insignificant with regard to the mechanical properties of the interface. Another study was conducted by Maenz and co-workers ${ }^{11}$ who subjected polyglycolic-co-lactic acid (PGLA) multifilaments to $\mathrm{O}_{2}$ plasma, followed by embedding them into $\mathrm{CaP}$ cement and measuring the interfacial shear strength using pull-out tests on these samples. This study also showed an increase in polymer hydrophilicity (water contact angle of $5^{\circ}$ ). Furthermore, a twofold increase in the interfacial shear strength of the treated filaments was measured. The increase was attributed to a combination of effects, namely, an increase in surface roughness of the multifilaments, increased wetting of the multifilaments with the cement, and the formation of chemical bonds between the calcium phosphates and the fibers.

The second mentioned approach, namely, the specific adsorption of a CaP component to the fiber surface, can be achieved by grafting an interlayer of specific cation attracting chelate groups onto the polymer surface. These groups can form a complex with divalent ions such as calcium $\left(\mathrm{Ca}^{2+}\right)$, which are highly available in a $\mathrm{CaP}$ cement precursor solution. The formation of such complexes then results in an increased nucleation and growth of $\mathrm{CaP}$ on surfaces containing such groups. In fact, in a study performed by Rautaray and co-workers, ${ }^{12}$ an increase in growth of $\mathrm{CaP}$ was observed on surfaces containing gold nanoparticles functionalized with aspartic acid (ASP) terminal groups. This growth effect is also attributed to chelating groups such as ASP, naturally present among others on silk fibroin in a study by Kong and co-workers ${ }^{13}$ and Schroeder and co-workers. ${ }^{14}$ The effects of these groups on the mechanical properties of $\mathrm{CaP}$ were reported by $\mathrm{Ou}$ and co-workers. ${ }^{15}$ They observed a twofold increase in compressive strength in $\mathrm{CaP}$ cement treated with silk fibroin solution, compared to untreated samples. This wet chemical approach is more specific regarding the deposited groups compared to plasma deposition. Also, surface roughening can often be reduced or avoided by choosing a suitable reaction.
The aim of this study was to demonstrate the grafting of ASP onto the surface of polymeric fibers, namely, PLA monofilaments to strengthen the PLA-CaP interface and analyze the effect of the treatment on both the adsorption of divalent ions and the mechanical properties of the PLA-CaP cement interface.

\section{EXPERIMENTAL SETUP AND METHODOLOGY}

PLA (Biomer L9000, Mm: $200000 \mathrm{kDa}$ ) was obtained from Biomer Biopolyesters (Krailing, Germany) in a bead form. A spool with a PLA monofilament with a diameter of $0.8 \mathrm{~mm}$ was kindly provided by Perlon Nextrusion AG (Bobingen Germany). The gases used for the plasma processes, i.e., argon, ethylene, and carbon dioxide, were of $99 \%$ purity and obtained from Carbagas (Gümligen, Switzerland). De-ionized water for the water vapor process was obtained from in-house supplies (18 M $\Omega$, Merck KGaA, Darmstadt, Germany). The consumables used in the wet chemistry process, i.e., aspartic acid, barium chloride dihydrate, disodium dihydrogen pyrophosphate, 1-ethyl-3-(3-dimethylaminopropyl) carbodiimide hydrochloride (EDC), ethylenediaminetetraacetic acid (EDTA), hexamethylene diamine (HMD), $N$-hydroxysuccinimide (NHS), nitrilotriacetic acid (NTA), and sodium hydroxide, were obtained from Merck KGaA (Darmstadt, Germany). HA nanoparticles were purchased from M K Impex Corp (Mississauga, Canada). Monocalcium phosphate monohydrate (Regent 12xx) was purchased from Innophos Inc. (Cranbury, USA). 2-( $N$-morpholino)-ethane sulphonic acid (MES) was obtained from Carl Roth $\mathrm{GmbH}+\mathrm{Co} . \mathrm{KG}$ (Karlsruhe, Germany). All solvents were obtained from commercial providers in ACS quality and were used without further purification.

\section{A. Sample manufacturing}

The polymer sample manufacturing consisted of different steps that are outlined schematically in Fig. 1.

\section{Polymer sample preparation}

To manufacture flat PLA films used for X-ray photoelectron spectroscopy (XPS) characterization tests [Fig. 1(a)], PLA beads were placed in a square steel frame of $1 \mathrm{~mm}$ thickness and an inner diameter of $100 \mathrm{~mm}$, which was covered from both sides with $0.2 \mathrm{~mm}$ thick aluminum foil. The form was then heated to $190^{\circ} \mathrm{C}$ for $20 \mathrm{~min}$, followed by an additional $20 \mathrm{~min}$ of cooling. The processed PLA films were then removed and placed in an aluminum foil for storage. The PLA monofilaments were cut into lengths of $80 \mathrm{~mm}$ using a surgical blade.

\section{Plasma treatment}

Both the flat PLA films and the PLA monofilaments [Fig. 1(b)] were coated using a low-pressure radio frequency $(\mathrm{RF}, 13.56 \mathrm{MHz})$ plasma reactor consisting of two plane-parallel electrodes separated by a glass ring $(30 \mathrm{~cm}$ in diameter, $5 \mathrm{~cm}$ in height). To establish a sufficiently high number of functional groups on the surface, protocols from Guex and co-workers ${ }^{16}$ and Guimond and co-workers ${ }^{17}$ were adapted for the current work and are described in the supplementary materials. The protocols were chosen such that minimal fiber degradation occurred. The finally selected protocol was as follows: RF power input of $60 \mathrm{~W}$; pressure of $0.1 \mathrm{mbar}$; process 

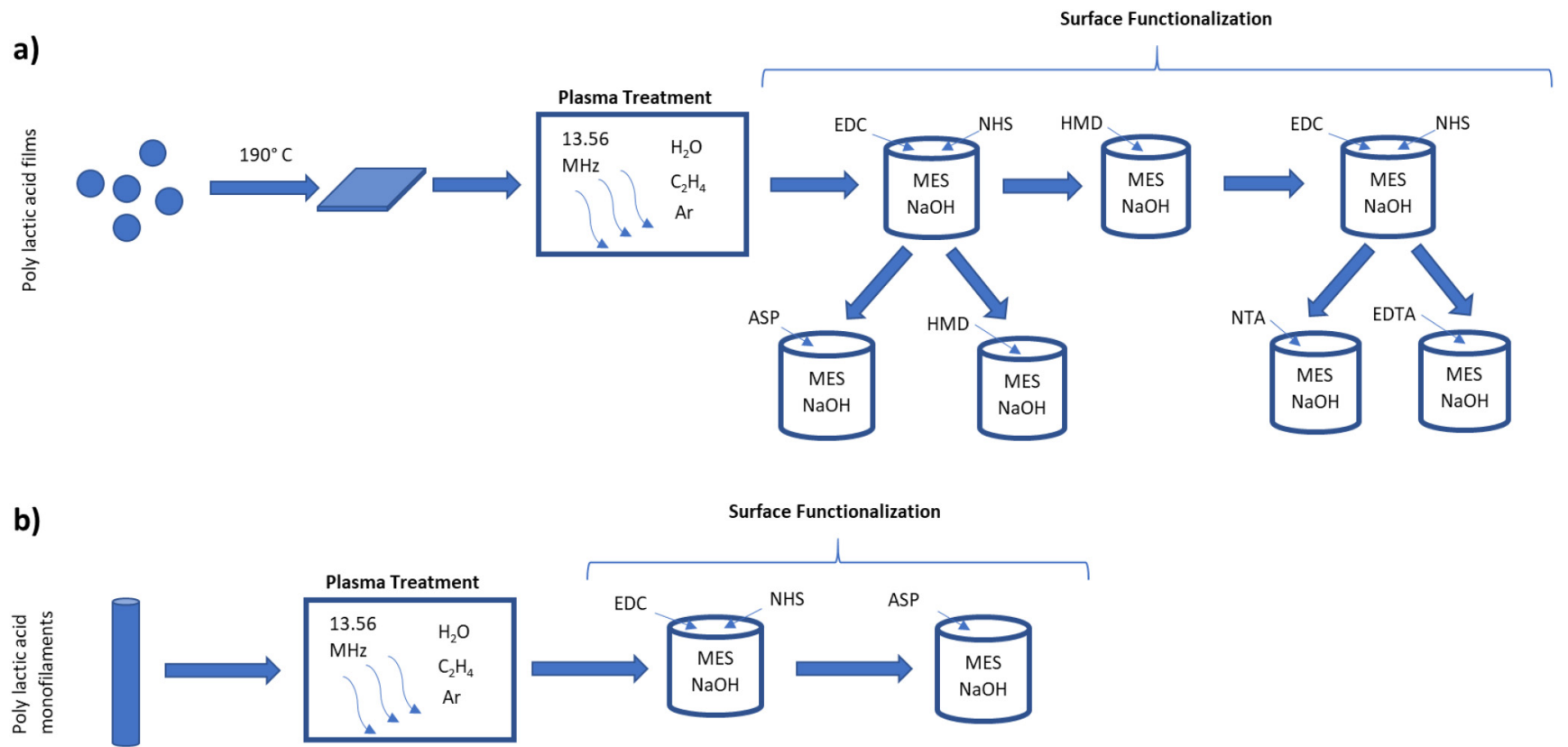

FIG. 1. Schematic of the functionalization treatment of (a) the PLA films and (b) the PLA monofilaments. The wet chemistry components, namely, the sodium hydroxide buffered 2-( $\mathrm{N}$-morpholino)ethanesulfonic acid solution (MES NaOH), as well as the reactants aspartic acid (ASP), 1-ethyl-3-(3-dimethylaminopropyl)carbodiimide (EDC), eEDTA, hexamethylenediamine (HMD), N-hydroxysuccinimide (NHS), and nitrilotriacetic acid (NTA), are shown.

duration of $5 \mathrm{~min}$; and a gas mixture of $36 \mathrm{SCCM} \mathrm{H}_{2} \mathrm{O}$ vapor, $6 \mathrm{SCCM} \mathrm{C}_{2} \mathrm{H}_{4}$, and 50 SCCM Ar. The latter was added to enhance crosslinking and stability of the deposited plasma polymer film.

After plasma treatment, the samples were placed in polyethylene bags filled with argon for transfer to further processing.

\section{Surface functionalization}

Following the plasma treatment, the prefunctionalized surfaces reacted with aspartic acid by carbodiimide chemistry using a protocol adapted from Edlund and co-workers. ${ }^{18}$ Each sample was placed in an individual well of a 24 -well plate and immersed in 0.1M MES$\mathrm{NaOH}$ buffer of $\mathrm{pH} 6$ for $30 \mathrm{~min}$ (reaction 1). Following that, two solutions, $0.2 \mathrm{M}$ of EDC and 0.5M NHS, were dissolved in the MES buffer (Carl Roth $\mathrm{GmbH}+\mathrm{Co}$. KG, Karlsruhe, Germany) added sequentially to the reaction and left to react under gentle shaking for $1 \mathrm{~h}$ (reaction 2). Afterward, the samples were washed and immersed in an MES buffer of $\mathrm{pH} 7$ and added to a solution of $0.05 \mathrm{M}$ of either HMD or aspartic acid (reaction 3). A subset of samples reacted with HMD was subjected again to reactions 1 and 2 and subsequently to a $0.05 \mathrm{M}$ solution of NTA or EDTA. The samples were left to react for $2 \mathrm{~h}$, and then removed, washed with de-ionized water, and dried under ambient conditions.

\section{Cement matrix engineering}

To manufacture the cement samples for the pull-out tests, an aqueous solution containing $0.06 \mathrm{M}$ disodium dihydrogen pyrophosphate (Sigma-Aldrich, Switzerland) was prepared with ultrapure water. This solution was mixed with $\beta$-tricalcium phosphate and monocalcium phosphate monohydrate powder $(16: 13 \mathrm{w}$ : $\mathrm{w}$ ratio) at a ratio of $3: 2$ powder to liquid (w:w). The resulting slurry was mixed for $2 \mathrm{~min}$ with a spatula to obtain a cementous paste, which was transferred into cylindrical forms of $20 \mathrm{~mm}$ in diameter and $30 \mathrm{~mm}$ in height. Immediately after, a PLA monofilament was embedded at a depth of $10 \mathrm{~mm}$ at the center of the paste. To ensure that the monofilament remained at the defined height and alignment throughout the setting process, it was fixed with a metal clip on the sample form. The resulting samples were left to set and cure for 3 days.

\section{B. Characterization}

\section{Surface functionalization}

The chemical surface analysis was conducted by XPS. To quantify the chelation efficacy, a subgroup of each sample type was immersed in an aqueous $0.1 \mathrm{M} \mathrm{BaCl}_{2}$ solution for $1 \mathrm{~h}$, subsequently rinsed with de-ionized water, and their barium content was quantified using XPS. Since calcium is highly abundant in the lab environment as a contaminant (e.g., supplementary material, Fig. 1), ${ }^{23}$ a similar but less commonly used element (barium) was chosen to minimize the risk of false positive results during the specific adsorption measurements. XPS measurements were performed either on a PHI 5000 VersaProbe II instrument (Minnesota, USA) or on an Axis Nova (Kratos Analytical, Manchester, United Kingdom). Both instruments were equipped with a monochromatic AlK $\alpha$ X-ray source. Detailed scans of the binding energies 
corresponding to $\mathrm{C} 1 \mathrm{~s}, \mathrm{O} 1 \mathrm{~s}, \mathrm{~N} 1 \mathrm{~s}$, and Ba3d were conducted. This was followed by survey scans to obtain an elemental overview and to quantify possible contaminations (example shown in the supplementary material, Figs. 1-7). ${ }^{23}$ Spectra were analyzed using CasaXPS software (Version 2.3.18, Casa software Ltd, GB). An iterated Shirley background was subtracted from the signals. The peak fitting was performed using a Gauss/Lorentz line shape, with the FWHM being set to be equal to that of the most dominant component in each scan. Charge correction was applied using a value of $285.0 \mathrm{eV}$ for the aliphatic C1s-peak.

\section{Wettability}

Hydrophilicity was quantified by measuring the static water contact angle with the sample surface on flat PLA films. To conduct the measurements, a contact angle meter SURFTENS-universal, OEG GmbH (Frankfurt, Germany) was used. The measurements were conducted as follows: de-ionized water was filled into a metered glass syringe, and a sessile water drop of $<10 \mu$ l volume was deposited on the surface of the sample using a syringe with a flat tip metal needle. The needle was then retracted, and the angle was measured on both sides of the droplet using SURFTENS 4.3 software. Three areas of each of the treatment groups were measured. Samples having contact angles below $45^{\circ}$ were considered as hydrophilic.

\section{Surface roughness}

Three PLA monofilaments from each group, i.e., control, plasma coating, and aspartic acid grafted samples, were analyzed for their surface roughness. Three randomly chosen areas of each sample were analyzed by white light interferometry using an $\mathrm{S}$ neox interferometer and confocal microscope (Sensofar, Barcelona, Spain). Prior to the measurement, the samples were blown with nitrogen to remove remaining particulate contaminants. The magnification was chosen to be $50 \times$ and the analyzed surface was $0.67 \times 0.26 \mathrm{~mm}$ along the monofilament. Three separate areas of each monofilament were analyzed. The data were analyzed using SensoMAP Premium (Version 7.4.8051, Digital Surf, France). From the topography, a polynomial function of the second degree corresponding to the cylindrical filament shape was derived and subtracted to allow a comparison with flat surfaces. The arithmetic mean roughness $R_{a}$ and waviness parameters were determined on profiles extracted from the topographies. The ratio of the real surface, i.e., the surface encompassing the three-dimensional contour of the sample accounting for the roughness, to projected surface, i.e., the two-dimensional top-down area, was determined to quantify the influence of the surface contribution of the sample roughness. These parameters were determined following the ISO 4287 and 4288 standards.

\section{Mechanical characterization of the interfacial strength}

The effect of the treatments on the integrity of the monofilaments was determined by measuring the ultimate tensile strength of untreated and treated monofilaments without embedding them into the $\mathrm{CaP}$ cement.
The stiffness and strength of the calcium phosphate bonding with the functionalized monofilaments were measured by conducting pull-out tests on monofilaments embedded in $\mathrm{CaP}$ cement. The interfacial modulus, which estimates the stiffness of the interfacial bond between the matrix and the monofilaments, was calculated as the value of the slope of the load-displacement curve within the bonded zone. Maximum debonding load was defined as the first maximum in load observed within the debonding zone. The debonding zone was defined as the zone between the start and the end of the interfacial debonding of the monofilament from the cement matrix. This was determined in the load-displacement curve between the point where the value of the slope starts to decrease and the inflection point where the sliding/friction zone starts (schemes describing typical pull-out behaviors are shown in the supplementary material, Fig. 19). ${ }^{23}$ This is adapted from the works of Zhan and Meschke ${ }^{19}$ and Friedrich and Wang ${ }^{20}$ who conducted numerical simulations of pull-out tests for applications in concrete.

The samples manufactured according to Sec. II A 4 were subjected to pull-out tests using a universal testing machine Zwicki-Line Z5.0 (Zwick GmbH \& Co. KG, Ulm, Germany) with a preload of $5 \mathrm{~N}$ and an embedding depth of $10 \mathrm{~mm}$ determined from a series of preliminary tests with untreated fibers. In all cases during the preliminary tests, linear load-displacement behavior was observed down to a minimum of $8 N$. Thus, $5 N$, a typical value used within the lab, was kept as a preload value for the following experiments. A speed of $2.5 \mathrm{~mm} / \mathrm{min}$ was chosen. After the maximum debonding load was measured, the experiment was stopped when the pull-out load reached $10 \%$ of the maximum measured strength of each sample. The monofilament groups used were as follows: (1) untreated as control, (2) plasma coated using protocol 3 (water vapor) described in Sec. II A 2, and (3) plasma treated with protocol 3 (water vapor) followed by a functionalization with aspartic acid using the protocols described in Sec. II A 3. At least four replicates were measured for each group. To test for polymer degradation during the treatment, five monofilaments from each group were subjected to tensile testing.

\section{Analysis of calcium adsorption on the filament surface after pull-out}

a. Postfracture image analysis. After the pull-out experiment, the extracted monofilaments were analyzed for $\mathrm{CaP}$ cement residue by stereomicroscopy using a Leica M205A (Leica Microsystems AG, Wetzlar, Germany). Areas with residue on the surface were observed as being matt areas under the white light of the microscope when compared to the shiny areas with low or no $\mathrm{CaP}$ content. The filaments were placed on a SEM sample holder such that the areas with the highest $\mathrm{CaP}$ concentration were visible for analysis. The samples were coated with $5 \mathrm{~nm}$ of palladium, using a sputter coater SCD 050 (Baltec, Pfäffikon, Switzerland), and analyzed using SEM. The magnification was chosen to be $150 \times$ at a working distance of $20 \mathrm{~mm}$ to allow full width coverage of the imaged filament part. The following areas were selected for analysis: (1) the bottom (tip) of the monofilament, being the deepest embedded area and subjected to the longest period of pull-out; (2) the top (stem), least embedded area and subjected to the shortest 
pull-out period; and (3) the center, containing the areas in between. At least four monofilaments of each group were analyzed.

b. Postfracture element analysis. The quantitative calcium content on different areas of the monofilaments was determined by analyzing the pulled-out samples with energy dispersive X-ray spectroscopy (EDX) using an Oxford instruments Xmax-N with the attached AzTec software version 3.3 (Oxford Instruments plc, Abingdon, United Kingdom). From the three locations determined in Sec. II A 4, at least four monofilaments of each sample were analyzed. The scan was done on an area of $750 \mu \mathrm{m}$ width $\times 560 \mu \mathrm{m}$ height corresponding to the observable diameter of the monofilament. A relative coverage of the area was then determined by image analysis. The images were then analyzed with Fiji bundle of imageJ version $1.52 \mathrm{n}$. The relative coverage was determined by counting the green pixels present in the images, which correspond to the areas where $\mathrm{Ca}(\mathrm{II})$ was detected.

\section{Statistical analysis}

All values reported are averages with their standard deviations. The statistical analysis was conducted using either one- or two-way ANOVA where appropriate. Additionally, a pairwise t-test with Bonferroni correction was conducted to determine significant differences among the different groups. The analysis was performed using R software, version 3.3.2, Python matplotlib library version 3.1.1 and Excel 2016. Principal component analysis was performed with the Analyse-It plugin for Excel, version 5.40.2. Probability values for rejecting the null hypothesis $(p)$ of $<0.05$ were considered as significant.

\section{RESULTS}

The functionalization and wettability analyses were conducted on PLA films with the following treatments: the untreated controls, water vapor plasma (Plasma), and the following functional groups functionalized to the polymer film surface using wet chemistry: ASP, HMD, NTA, and EDTA, while the surface roughness, mechanical, and postfracture analyses were conducted on PLA monofilaments with the following treatments: the untreated controls, Plasma, and ASP.

\section{A. Surface functionalization}

Analysis of different element concentrations showed that the adsorption of divalent barium from aqueous solution was the highest for ASP (6.4 \pm 0.2 at. \%), followed by control (4.2 \pm 0.7 at. \%) and EDTA $(2.9 \pm 0.4)$ (see Table I and supplementary material, Table 2) ${ }^{23}$ For C1s, Plasma had the highest concentration of $\mathrm{C}$, followed by control and EDTA (73.0 \pm 0.6 at. \%, $72.2 \pm 0.4$ at. \%, and $64.0 \pm 3.3$ at. \%) [Table I and supplementary material, Figs. 1-3, Table 2]. ${ }^{23}$ Analysis of the O1s spectrum (Table I and supplementary material, Figs. 4-6, Table 2) ${ }^{23}$ showed that HMD and NTA contained the highest oxygen concentration $(34.9 \pm 0.3$ at. $\%$ and $34.5 \pm 1.2$ at. \%), followed by EDTA group (30.8 \pm 2.5 at. \%). Low concentrations of residual chloride $(<1$ at. \%) stemming from the barium chloride salt as well as silicon (glassware), sulfur, and sodium (buffering solution) were measured in all samples.

\section{B. Wettability}

The lowest static water contact angle corresponding to the highest hydrophilicity was measured after treatment with EDTA $\left(32^{\circ} \pm 10^{\circ}\right)$, and the highest value corresponding to the lowest hydrophilicity was measured for the control $\left(77^{\circ} \pm 6^{\circ}\right)$.

\section{Surface roughness}

The quantitative analysis by interferometry (Fig. 2) measured along the length axis of the monofilament showed an $R_{a}$ of $45 \pm 5 \mathrm{~nm}$ for the control group, $134 \pm 34 \mathrm{~nm}$ for the Plasma, and $93 \pm 26 \mathrm{~nm}$ for the ASP in pull-out direction. Parallel grooves stemming from the monofilament manufacturing process were observed on all monofilaments. Perpendicular to the monofilament length axis, the $R_{a}$ values were $74 \pm 15 \mathrm{~nm}$ for the control, $210 \pm 45 \mathrm{~nm}$ for the Plasma, and $84 \pm 26 \mathrm{~nm}$ for the ASP. The ratios of real to projected surfaces were as follows: $1.005 \pm 0.002$ for control, $1.034 \pm 0.013$ for Plasma, and $1.008 \pm 0.001$ for ASP.

\section{Mechanical characterization of the interfacial strength}

The tensile tests showed that all the monofilaments had a tensile strength of $70 \pm 3 \mathrm{MPa}$ regardless of the treatment they received.

TABLE I. Overview of the different measurement results performed in this study.

\begin{tabular}{|c|c|c|c|c|c|c|c|}
\hline \multirow[b]{2}{*}{ Sample } & \multicolumn{3}{|c|}{ Concentration (at. \%) } & \multirow[b]{2}{*}{ Contact angle } & \multicolumn{2}{|c|}{ Pull-out tests } & \multirow{2}{*}{$\begin{array}{c}\text { EDX } \\
\text { measurements } \\
\begin{array}{c}\text { Calcium surface } \\
\text { coverage }(\%)\end{array}\end{array}$} \\
\hline & Carbon & Oxygen & Barium & & $\begin{array}{l}\text { Max. debond. } \\
\text { load }(\mathrm{N})\end{array}$ & $\begin{array}{l}\text { Interfacial stiffness } \\
(\mathrm{N} / \mathrm{mm})\end{array}$ & \\
\hline Control & $72.2 \pm 0.4$ & $21.2 \pm 0.8$ & $4.2 \pm 0.7$ & $77^{\circ} \pm 6^{\circ}$ & $13 \pm 4$ & $12 \pm 4$ & $11 \pm 9$ \\
\hline Plasma & $73.0 \pm 0.6$ & $23.1 \pm 0.6$ & $2.1 \pm 0.5$ & $71^{\circ} \pm 8^{\circ}$ & $12 \pm 5$ & $16 \pm 10$ & $17 \pm 10$ \\
\hline HMD & $61.5 \pm 1.3$ & $34.9 \pm 0.3$ & $2.1 \pm 1.0$ & $42^{\circ} \pm 5^{\circ}$ & & & \\
\hline ASP & $60.6 \pm 1.6$ & $30.4 \pm 1.1$ & $6.4 \pm 0.2$ & $42^{\circ} \pm 5^{\circ}$ & $17 \pm 5$ & $23 \pm 7$ & $29 \pm 17$ \\
\hline NTA & $63.3 \pm 1.0$ & $34.5 \pm 1.2$ & $1.2 \pm 0.1$ & $36^{\circ} \pm 6^{\circ}$ & & & \\
\hline EDTA & $64.0 \pm 3.3$ & $30.8 \pm 2.5$ & $2.9 \pm 0.4$ & $32^{\circ} \pm 10^{\circ}$ & & & \\
\hline
\end{tabular}



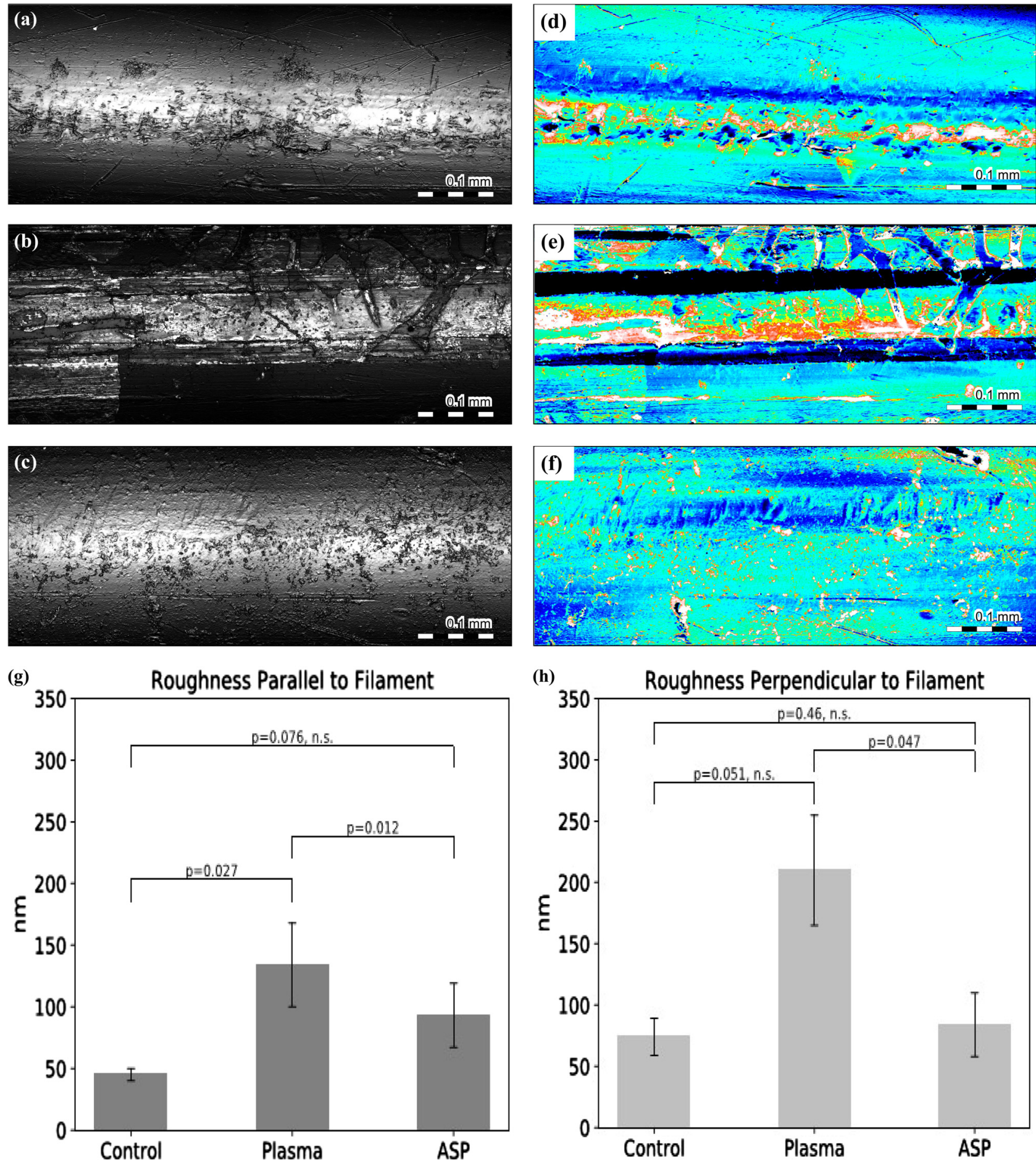

FIG. 2. Optical image at $50 \times$ magnification of the monofilaments (a) control, (b) Plasma, and (c) ASP. Color plot of the topography of the monofilaments (d) control, (e) Plasma, and (f) ASP. The darker colors (blue/violet) denote the valleys (indicated exemplarily with solid arrows), and the lighter colors (yellow red white) denote the peaks of the surface (indicated exemplarily with dotted arrows). Bar plots of the surface roughness of the filaments parallel $(\mathrm{g})$ and perpendicular ( $\mathrm{h}$ ) to the filament axis; $\mathrm{n} . \mathrm{s}$. indicates nonsignificance. 

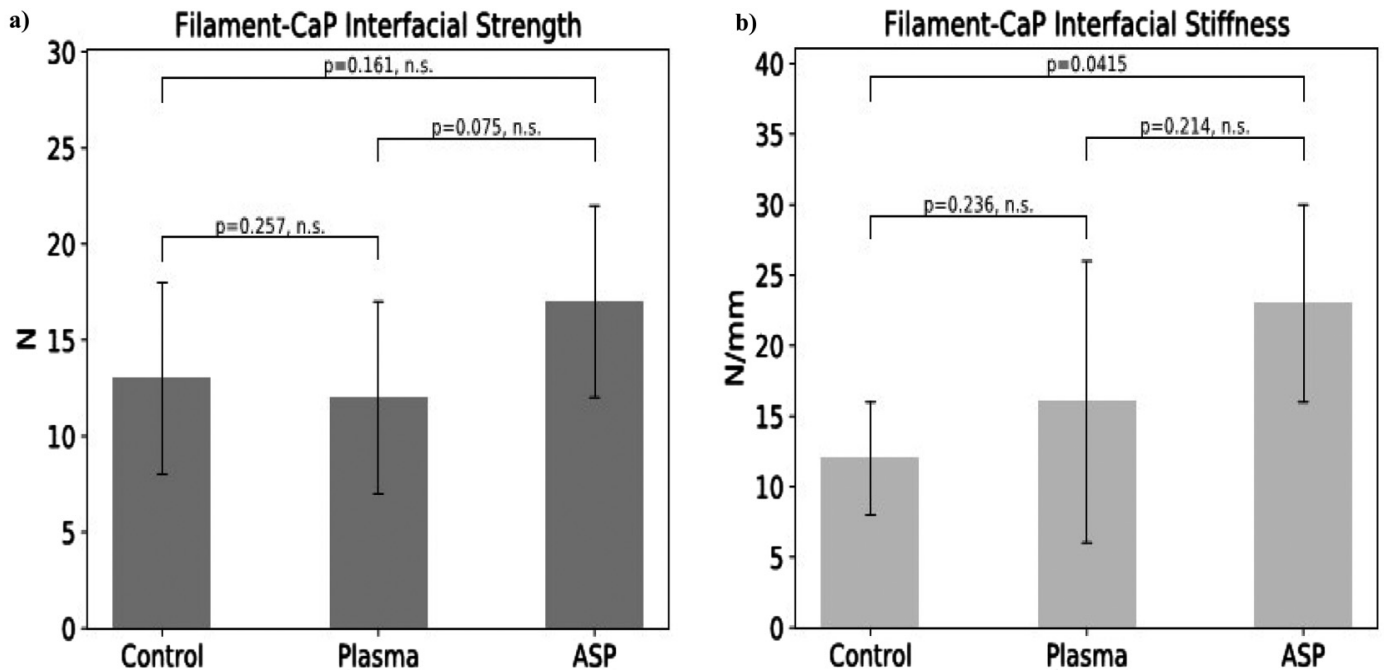

FIG. 3. Bar plots of the interfacial strength (a) and stiffness (b) of the different treatments. n.s. indicates nonsignificance.

The interfacial stiffness values measured were $12 \pm 4,16 \pm 10$, and $23 \pm 7 \mathrm{~N} / \mathrm{mm}$ for the control, Plasma, and ASP group, respectively. The differences between control and ASP were significant $(\mathrm{p}=0.03)$. The values for maximum debonding load were $13 \pm 2$, $12 \pm 5$, and $17 \pm 5 \mathrm{~N}$ (Table I, Fig. 3) for the control, Plasma, and ASP group, respectively. The maximum debonding loads measured showed a broad scattering with the differences being not significant $(\mathrm{p}=0.27$ ). Debonding occurred generally within $1 \mathrm{~mm}$ (approximately $10 \%$ ) of displacement. One filament (CTRL 5) of the control group was embedded approximately $1.5 \mathrm{~mm}$ deeper (supplementary material, Fig. 20a $)^{23}$ due to a measurement error; however, its debonding behavior was in line with the other samples and was, therefore, included in the analysis. The debonding zone width varied between a sharp peak (no discernible debonding zone) and approximately $2 \mathrm{~mm}$ in displacement among the samples. A majority of the samples in all groups showed slip hardening behavior beyond the debonding zone (supplementary material, Figs. 19 and 20). ${ }^{23}$

\section{E. Calcium adsorption on the filament surface after pull-out}

The control samples had a relative coverage area of $\mathrm{Ca}(\mathrm{II})$ of $11 \pm 7 \%$ of the total area analyzed, Plasma had an average of $17 \pm 10 \% \mathrm{Ca}$ (II), and ASP showed an average of $29 \pm 17 \% \mathrm{Ca}$ (II), (Table I). The coverage of calcium was significantly higher $(p=0.002)$ between the ASP and the control group. No significant difference was found between ASP and Plasma $(p=0.054)$ or Plasma and the control group $(\mathrm{p}=0.08)$ regarding calcium coverage (Fig. 4). No significant difference in calcium concentration was observed with regard to the position of the measurement in the sample (top/bottom/center) ( $\mathrm{p} \geq 0.69)$.

\section{DISCUSSION}

\section{A. Surface functionalization}

The observed $50 \%$ increase in retained barium on the ASP treated surface after rinsing, compared to control, agrees with the
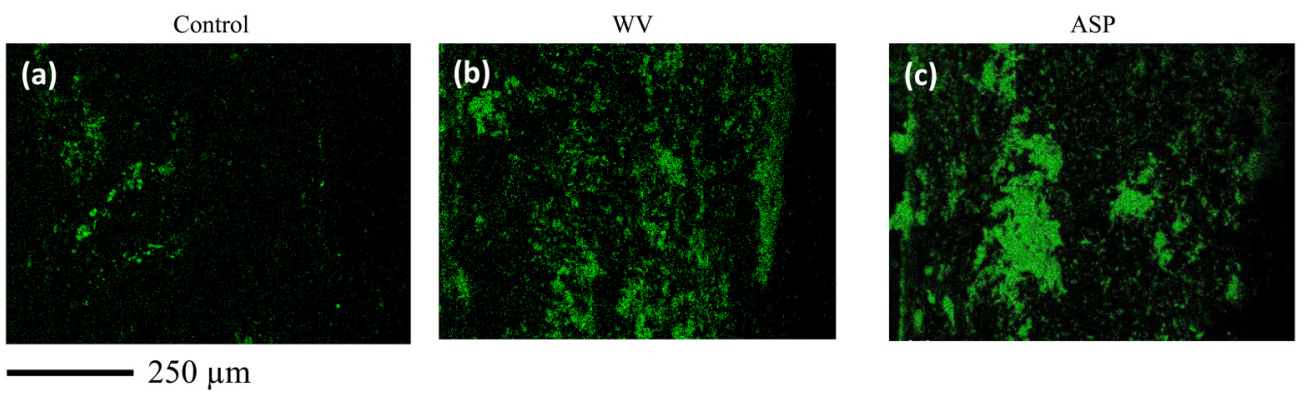

FIG. 4. EDX images of the distribution of calcium (green) on different PLA monofilament areas after pull-out testing. Untreated control (a), water vapor plasma treated (Plasma, b), and functionalized with aspartic acid (ASP, c); different amounts of calcium phosphate are visible as green areas on the monofilament surface. 
results obtained by Rautaray and co-workers. They observed a strong bonding between the divalent calcium ions and ASP coated gold nanoparticles. ${ }^{12}$ The ratio of calcium ions adsorbed from solution can be assumed to be similar to the ratio of adsorbed barium due to the same mechanism of adsorption. The increased oxygen to carbon ratio in the sample suggests an increased presence of carboxyl groups stemming from the ASP and carbonyl groups caused by unreacted NHS. A lower barium and a higher oxygen concentration were measured in the HMD, NTA, and EDTA groups compared to ASP. This suggests that a higher reaction yield of chelating groups was obtained in ASP compared to all other groups. This is due to the additional reaction step required to attach the groups to the surface, resulting in an overall lower concentration of chelating groups on the surface and a higher concentration of retained NHS. Regarding the reaction efficiency, both ASP and NTA were present in bidentate form, where the third arm of NTA was linked to the surface. However, approximately a fivefold concentration of barium was present on the ASP samples compared to the NTA; it can, therefore, be assumed that five times more ASP was present on the surface compared to NTA. Assuming a similar reaction yield of NTA and EDTA, the EDTA covered surface had approximately a twofold concentration of barium. Since ETDA is present in the tridentate form, with the fourth arm linked to the surface, it can be assumed that EDTA had double the chelation strength of NTA and ASP. Thus, ASP presents a compromise between reaction efficiency and chelation strength. The reason for low concentration of barium on the Plasma samples compared to control, despite the increase in oxygen concentration, is unclear. It is possible that the carbonyl groups were not in a chelating, i.e., bidentate, form, thus providing less sites for barium to remain adsorbed after the rinsing step. The results from the functionalization step showed different residual elements stemming from the glassware and buffering solution. The presence of these residuals suggests a need for an optimized rinsing procedure that at the same time does not damage the coating.

\section{B. Hydrophilicity}

All functionalized samples (ASP, HMD, NTA, and EDTA) showed distinctly reduced water contact angles. However, this increase in surface hydrophilicity did not result in an increase in barium concentration on the surface of all groups. In fact, the two groups with the lowest water contact angle, namely EDTA and NTA, had a lower barium concentration than the control group. On the other hand, ASP, with a lower hydrophilicity than the above groups, had double the concentration of barium compared to the other groups. This supports the argument that the bond between the ASP and the barium discussed in Sec. IV A had a higher effect on barium retention than hydrophilicity alone. Indeed, the principal component analysis performed on control, Plasma, and ASP (supplementary material, Table 3$)^{23}$ showed that the highest contribution for the first component (proportion of 0.671 ) came from the chelation (0.584). Both surface roughness parallel and perpendicular contributed negatively $(-0.456,-0.557)$ as well as hydrophilicity, but to a smaller degree $(-0.377)$. Furthermore, plotting hydrophilicity against the barium concentration (supplementary material, Fig. 23) ${ }^{23}$ shows the effect of ASP as outlier against the rest of the data. The hydrophilicity of the Plasma samples, i.e., by depositing a functional plasma polymer layer, was comparable to other typical plasma treatments used to increase hydrophilicity. For example, the PGLA samples treated with $\mathrm{O}_{2}$ plasma by Canal and co-workers, ${ }^{10}$ Guimond and co-workers, ${ }^{17}$ or Körner and co-workers ${ }^{21}$ achieved angles approximately $20^{\circ}$ lower with their protocols than the Plasma samples considered here. This difference might be ascribed to the addition of Ar in the plasma helping to stabilize the nanoscaled plasma polymer film via crosslinking at the expense of polar functional groups. Körner and co-workers reported angles below $10^{\circ}{ }^{21}$ However, the surface layer treated in such a way dissolved in aqueous media. This would not be useable when mixing with a cementeous paste, which is an aqueous, generally acidic medium. Therefore, to be of use in $\mathrm{CaP}$ cements, the optimized, functional plasma polymer coating as a precursor step should be selected such that it remains stable in an aqueous medium. It was, thus, concluded that a decreased water contact angle did not influence the adsorption or retention of divalent species, and that the chelating effect had a stronger effect on the adsorption and retention of divalent species than increasing hydrophilicity. For that reason, the following three groups (1) ASP functionalization, and its precursor step (2) water vapor plasma coating, and (3) the unreacted control were used for further testing.

\section{Surface roughness}

The origin of the roughness observed can be attributed to two factors: (1) The fiber production process (filament extrusion), visible as grooves parallel to the pull-out direction (Fig. 2). The plasma treatment that resulted in an overall higher roughness both parallel and perpendicular to the pull-out direction. The effect of the surface treatments on the overall surface roughness was minimal, corresponding to a $3 \%$ increase in the real surface of the Plasma group compared to the control. This was despite the observed twofold increase in $\mathrm{R}_{\mathrm{a}}$ in the pull-out direction, and the threefold increase perpendicular to the pull-out direction. The surface increase of the ASP group compared to control was lower by an order of magnitude $(+0.3 \%)$. The $R_{a}$ values reported in this manuscript were between 45 and $210 \mathrm{~nm}$ that would agree with the increase in roughness reported after $\mathrm{O}_{2}$ plasma for $10 \mathrm{~min}$ treatment by Maenz and co-workers for PGLA surfaces ${ }^{11}$ and Canal and co-workers on PLA $^{10}$ after 5 min of treatment. These $R_{a}$ values were seemingly below $200 \mathrm{~nm}$ and below $1 \mu \mathrm{m}$, respectively. The decrease in surface roughness of the ASP group following the plasma reaction could be due to a partial dissolution or detachment of coated areas and/or their edges from the plasma reaction during the wet chemical functionalization step that was conducted in an aqueous solution.

\section{Mechanical characterization of the interfacial strength}

The analysis of interfacial modulus showed that the ASP group had, with a twofold increase compared to control, the highest values within all groups. The Plasma group showed a broad scattering and was not significantly different compared to the control or ASP group. It can be argued that the ASP coating resulted in a stiffer bonding between the CaP and the PLA that stems from the increased bonding between the ASP and the Ca ions as discussed in Sec. IV A. Regarding the interfacial strength, no significant difference was observed between the groups, which also showed a broad scattering 
of the data. This is a typical behavior often observed in cements such as concrete. ${ }^{22}$ Due to their brittle and polycrystalline nature, meaning that the defect distribution during the setting procedure within the samples has a large effect on their the behavior under load, a large variance between the samples occurs, even under identical manufacturing conditions. It was also observed that increased monofilament $\mathrm{R}_{\mathrm{a}}$ that was induced by the plasma treatment did not have an impact on the mechanical properties, a fact already reported in previous studies. ${ }^{10,11}$ Despite the large variances, the results with regard to interfacial strength can be considered consistent with what Canal and co-workers observed, ${ }^{10}$ namely that the flexural strength did not increase beyond that of the untreated cement matrix. This finding and the partial coverage of the monofilaments with $\mathrm{CaP}$ suggest that the matrix and the interface failed at similar strengths and that part of the $\mathrm{CaP}$ initially attached to the surface was removed during pull-out. The results of the interfacial strength correspond to the fracture strength of the cement matrix. This is consistent with the slip hardening in the sliding/friction phase, which was observed on most of the pull-out tested samples. Such slip hardening can stem from a residual $\mathrm{CaP}$ remaining on the monofilaments during pull-out, resulting in an increased friction due to an increased surface of the pulled-out monofilament from residual $\mathrm{CaP}$.

\section{E. Postfracture calcium adsorption}

The results of the postfracture analysis showed an almost threefold increase in calcium concentration for the ASP group, compared to control. This increase was also reported in the literature $^{11}$ for plasma treated PGLA and PLA multifilaments. This increase was reported to be originating from both an increase in hydrophilicity, resulting in an increased wetting of the filament surface with $\mathrm{CaP}$ cement paste, and an increase in $\mathrm{R}_{\mathrm{a}}$ resulting in an increase of possible nucleation sites for the CaP. In this work, however, $\mathrm{R}_{\mathrm{a}}$ did not show a significant influence; therefore, the effects from the surface chemistry were dominant. The difference between ASP and Plasma was nonsignificant, seemingly due to the broad scattering observed between the individual samples as discussed previously in Sec. IV D. No significant difference in calcium concentration was measured between the different areas of the monofilament, which is an indication for a similar distribution of the cement along the fiber surface.

\section{SUMMARY AND CONCLUSION}

We demonstrated the successful grafting of aspartic acid groups onto the surface of PLA monofilaments, which resulted in a $50 \%$ increase in adsorption and retention of divalent elements on the surface using barium as exemplary element and in an almost threefold increase in calcium coverage observed postfracture. This treatment resulted in a twofold increase in interfacial stiffness between the PLA monofilament and the CaP matrix. No significant increase in mechanical interfacial strength of the interface was observed, apparently due to the weakness of the CaP matrix. The minimal increase in the real surface of the monofilaments following plasma treatments revealed that the use of monofilaments allows for a good comparability between the groups.

\section{ACKNOWLEDGMENTS}

This work was partially supported by a grant from the Swiss National Research Program "Opportunities and Risks of Nanomaterials" NRP 64, Grant No. 406440_131273. The authors would like to acknowledge Thomas Niessen from Empa and Stefan Röthlisberger and Olivier Loeffel from RMS Foundation for their help with plasma processing, pull-out testing, and XPS analysis and Reto Luginbuehl from RMS Foundation for his helpful ideas, comments, and corrections regarding this manuscript.

\section{REFERENCES}

${ }^{1}$ N. Döbelin, R. Luginbühl, and M. Bohner, Chimia 64, 723 (2010).

${ }^{2}$ H. H. K. Xu, F. C. Eichmiller, and A. A. Giuseppetti, J. Biomed. Mater. Res. 52, 107 (2000).

${ }^{3}$ B. Pukánszky, Eur. Polym. J. 41, 645 (2005).

${ }^{4}$ F. Libonati, A. K. Nair, L. Vergani, and M. J. Buehler, Mech. Res. Commun. 58, 17 (2014).

${ }^{5}$ Y. You, B. M. Min, S. J. Lee, T. S. Lee, and W. H. Park, J. Appl. Polym. Sci. 95, 193 (2005).

${ }^{6}$ I. Engelberg and J. Kohn, Biomaterials 12, 292 (1991).

${ }^{7}$ Y. Zuo, F. Yang, J. G. C. Wolke, Y. Li, and J. A. Jansen, Acta Biomater. 6, 1238 (2010).

${ }^{8}$ A. A. Leal, J. C. Veeramachaneni, F. A. Reifler, M. Amberg, D. Stapf, G. A. Barandun, D. Hegemann, and R. Hufenus, Mater. Des. 93, 334 (2016).

${ }^{9}$ N. Graupner, K. Albrecht, D. Hegemann, and J. Müssig, J. Appl. Polym. Sci. 128, 4378 (2013).

${ }^{10}$ C. Canal, S. Gallinetti, and M. P. Ginebra, Plasma Process. Polym. 11, 694 (2014).

${ }^{11}$ S. Maenz, M. Hennig, M. Mühlstädt, E. Kunisch, M. Bungartz, O. Brinkmann, J. Bossert, R. W. Kinne, and K. D. Jandt, J. Mech. Behav. Biomed. Mater. 57, 347 (2016).

${ }^{12}$ D. Rautaray, S. Mandal, and M. Sastry, Langmuir 21, 5185 (2005).

${ }^{13}$ X. D. Kong, F. Z. Cui, X. M. Wang, M. Zhang, and W. Zhang, J. Cryst. Growth 270, 197 (2004).

${ }^{14}$ W. A. Schroeder, L. M. Kay, B. Lewis, and N. Munger, J. Am. Chem. Soc. 77, 3908 (1955).

${ }^{15}$ J. Ou, Y. Jiang, and Z. Zhang, Adv. Mater. Res. 343-344, 882 (2012).

${ }^{16}$ A. G. Guex, D. Hegemann, M. N. Giraud, H. T. Tevaearai, A. M. Popa, R. M. Rossi, and G. Fortunato, Colloids Surf. B 123, 724 (2014).

${ }^{17}$ S. Guimond, B. Hanselmann, M. Hossain, V. Salimova, and D. Hegemann, Plasma Processes Polym. 12, 328 (2015).

${ }^{18}$ U. Edlund, T. Sauter, and A. C. Albertsson, Polym. Adv. Technol. 22, 2368 (2011).

${ }^{19} \mathrm{Y}$. Zhan and G. Meschke, paper presented at the 8th International Conference on Fracture Mechanics of Concrete and Concrete Structures FraMCoS-8, Toledo, Spain, 10-14 March, 2013 (unpublished).

${ }^{20}$ L. F. Friedrich and C. Wang, Lat. Am. J. Solids Struct. 13, 1937 (2016).

${ }^{21}$ E. Körner, G. Fortunato, and D. Hegemann, Plasma Processes Polym. 6, 119 (2009).

${ }^{22}$ B. A. Proctor, Cem. Concr. Compos. 12, 53 (1990).

${ }^{23}$ See supplementary material at https://doi.org/10.1116/1.5129989 for detailed XPS survey scans, stress- strain curves, sessile drop figures and statistical analysis. 